\title{
Avaliação dos efeitos do amitraz por via epidural em vacas
}

Ricardo Miyasaka de ALMEIDA $^{1}$

Carlos Augusto de Araújo

VALADÃO'

Anderson FARIAS 1

Juan Carlos Moreno

DUQUE ${ }^{2}$

Adriana Helena de SOUZA²

Correspondência para:

RICARDO MIYASAKA DE ALMEIDA

Departamento de Medicina Veterinária

Faculdade de Medicina Veterinária

Campus II - UPIS

SEPS, 712/912, conj. A, Asa Sul

70390-125 - Brasília - DF

ricardo02608@upis.br

Recebido para publicação: 23/10/2003 Aprovado para publicação: 10/11/2005

1- Departamento de Medicina Veterinária da Faculdade de Medicina Veterinária, Campus II - UPIS, Brasília - DF

2- Departamento de Clínica e Cirurgia Veterinária da Faculdade de Ciências Agrárias e Veterinárias da UNESP, Campus de Jaboticabal, Jaboticabal - SP

\section{Resumo}

O amitraz possui efeitos sistêmicos resultantes da atividade intrínseca com receptores adrenérgicos do tipo a-2. Nesse estudo, avaliou-se os efeitos das injeções epidurais de amitraz $(0,1$ e 0,4 mg.kg-1) ou DMSO 10\% (5,0 mL) sobre a freqüência cardíaca (FC), pressão arterial sistólica (PAS), freqüência respiratória (FR), motilidade ruminal (MR), temperatura retal (TR), altura de cabeça (AC) e as latências da resposta a estímulos nociceptivos nas regiões da coxa (LECC) e coroa do casco (LRRM) de vacas. Houve uma diminuição das variáveis $\mathrm{FC}$ e MR nos animais que receberam amitraz. Não houve diferenças significativas na FR, PAS e AC entre os grupos estudados. A LECC e a LRRM foram maiores no grupo amitraz $\left(0,4 \mathrm{mg} \cdot \mathrm{kg}^{-1}\right)$ entre 25 e 120 minutos. Concluiu-se que o amitraz aumenta a latência de resposta a estímulo nociceptivo, sem induzir efeitos colaterais sistêmicos severos nas doses utilizadas, por via epidural em vacas.

\section{Introdução}

Os agonistas de receptores a-2 adrenérgicos (agonistas a-2) são substâncias derivadas da tiazina que causam sedação, miorrelaxamento e analgesia dose-dependentes, sendo comumente empregados como sedativos, analgésicos e na medicação préanestésica ${ }^{1}$. A administração epidural dos agonistas a-2 é amplamente descrita na literatura em diversas espécies, tendo efeitos característicos como sedação, antinocicepção, ataxia (de baixa incidência) e maior duração e potência, quando comparados ao uso de anestésicos locais ${ }^{2,3,4,5}$. Os principais efeitos sistêmicos relacionados ao uso epidural desses fármacos, em bovinos, são bradicardia, ligeira hipotensão, inibição da motilidade ruminal e timpanismo $0^{5,6,7}$.

O amitraz é um derivado formamidínico utilizado na Medicina Veterinária para o tratamento de ectoparasitoses em pequenos e grandes animais ${ }^{8}$. Estudos farmacodinâmicos e de toxicidade conduzidos em ratos, cães, gatos e pôneis, demonstraram que o amitraz apresenta atividade intrínseca em receptores a-2 adrenérgicos centrais e periféricos ${ }^{9,10,11}$. Em eqüinos, o amitraz injetado por via epidural, na dose de $0,1 \mathrm{mg} \cdot \mathrm{kg}^{-1}$, causou sedação e relaxamento muscular, com mínimos efeitos deletérios nos sistemas cardiovascular e respiratório ${ }^{12}$. $\mathrm{Na}$ espécie bovina, a administração intravenosa de 0,4 mg. $\mathrm{kg}^{-1}$ dessa substância promoveu bradicardia e diminuição na motilidade ruminal, além de antinocicepção semelhante àquela obtida com uma dose de 0,1 mg.kg${ }^{1}$ de xilazina ${ }^{13}$, enquanto que o seu emprego como medicação pré-anestésica antes da indução anestésica de bezerros com cetamina, mostrou efeitos semelhantes aos observados nos animais pré-tratados com xilazina ${ }^{14}$.

O dimetil sulfóxido (DMSO) vem sendo utilizado na Medicina Veterinária como veículo para diversos medicamentos, 
pois possui propriedade de carrear consigo substâncias de pequeno peso molecular. $\mathrm{O}$ DMSO também possui outras aplicações, como estimulador da cicatrização, antiinflamatório, antioxidante e analgésico, e ainda demonstrou ser uma substância com baixa toxicidade ${ }^{1,15}$. O amitraz, por ser um composto insolúvel em água, foi diluído em DMSO conforme algumas citações da literatura, as quais demonstraram além da compatibilidade, a inexistência de sinergismo entre as duas substâncias ${ }^{3,12,16}$.

O objetivo desse estudo foi avaliar os possíveis efeitos cardiorespiratórios, comportamentais e antinociceptivos do amitraz após sua administração epidural, nas doses de 0,1 e 0,4 mg.kg-1 em vacas.

\section{Materiais e Métodos}

Foram utilizadas 21 fêmeas bovinas, da raça holandesa, adultas e saudáveis, pesando entre 452 e $655 \mathrm{~kg}(553,50 \pm 143,54$ $\mathrm{kg})$. Durante a fase experimental, os animais foram mantidos em pasto e alimentados com silagem de milho, água à vontade $\mathrm{e}$ suplementados com sal mineral. Todos os procedimentos e avaliações foram realizados em um tronco de contenção, em local tranqüilo e climatizado, com temperatura média de $25^{\circ} \mathrm{C}$. Os animais foram distribuídos aleatoriamente em 3 grupos, denominados grupo amitraz $0,1 \quad(\mathrm{~N}=8)$, grupo amitraz $0,4(\mathrm{~N}=8)$ e grupo controle $(\mathrm{N}=5)$, os quais receberam por via epidural, $0,1 \mathrm{mg} \cdot \mathrm{kg}^{-1}$ e $0,4 \mathrm{mg} \cdot \mathrm{kg}^{-1}$ de amitraz (Amitraz técnico - Laboratório Sintesul S.A., Pelotas, RS) diluído em DMSO (DMSO, P.A. Quimibrás Indústrias Químicas S.A., Rio de Janeiro, RJ) $10 \%$ e 5,0 mL de DMSO 10\%, respectivamente. $\mathrm{O}$ volume final das soluções de amitraz foi ajustado para 5,0 $\mathrm{mL}$ com água destilada, para padronização do volume de acordo com literatura ${ }^{2,5,6,7,17}$. A injeção das soluções foi realizada no canal epidural, em região correspondente ao espaço entre a quinta vértebra sacral $\left(S_{5}\right)$ e a primeira vértebra coccígea $\left(\mathrm{Co}_{1}\right)$, o qual foi identificado pela movimentação da cauda no sentido dorso-ventral, associada à palpação da depressão entre as vértebras citadas. Após a localização do espaço entre $\mathrm{S}_{5}-\mathrm{Co}_{1}$, efetuouse a tricotomia e anti-sepsia da região com álcool iodado. Em seguida, realizou-se um botão anestésico subcutâneo com $2,0 \mathrm{~mL}$ de cloridrato de lidocaína a $2 \%$ com vasoconstritor (Anestésico Bravet Laboratório Bravet Ltda., Rio de Janeiro, RJ). Decorridos 20 minutos da anestesia local, uma agulha espinhal de Tuohy (Agulha técnica reusável de Tuohy 80x10F - Becton Dickinson Indústrias Cirúrgicas Ltda., Juiz de Fora, $M G$ ) foi introduzida no espaço $S_{5}$ $\mathrm{Co}_{1}$ até atingir o canal epidural. $\mathrm{O}$ correto posicionamento da agulha foi confirmado pela ausência de resistência à injeção de 1,0 $\mathrm{mL}$ de ar, associado ao teste da gota pendente. Os fármacos foram aplicados na razão de 1,0 $\mathrm{mL}$ a cada 3,0 segundos.

Antes dos tratamentos, os animais foram avaliados por duas vezes, com um intervalo de dez minutos, para que fossem registradas as variáveis fisiológicas basais (T0) de cada animal para: freqüência respiratória (FR), por observação da movimentação do gradil costal durante 1 minuto; freqüência cardíaca (FC), medida por oximetria de pulso $^{\mathrm{V}}$, com o sensor infravermelho posicionado na teta ou na vulva do animal; temperatura retal (TR), mensurada por termômetro clínico de mercúrio, introduzido na ampola retal, durante 3 minutos; movimentos ruminais (MR), por meio de auscultação com estetoscópio, posicionado na fossa paralombar esquerda, durante 5 minutos; e pressão arterial sistólica (PAS), por meio de método não-invasivo, empregandose aparelho de ultra-sonografia por sistema Doppler (Ox-P-10 EMAI - TRANSMAI Equipamentos Médico Hospitalares Ltda., São Paulo, SP), com manguito posicionado na base da cauda do animal e o transdutor ultra-sônico fixado na porção ventral da cauda, sobre a artéria coccígea média, distalmente ao manguito.

As variáveis fisiológicas acima citadas foram aferidas novamente aos 5,10 e 15 minutos (T5, T10, T15) após as injeções 
epidurais, passando a intervalos de 10 minutos até os 75 minutos (T25,.., T75), posteriormente a cada 15 minutos até os 120 minutos (T120) e, finalmente, com intervalos de 30 minutos até os 360 minutos (T150,..., T360). As variáveis MR e TR foram avaliadas a cada 15 minutos, até o final do período de observação.

Paralelamente, realizou-se a avaliação comportamental dos animais nos mesmos intervalos definidos para os exames clínicos, para a observação de possível sedação e antinocicepção. O grau de sedação foi estimado mensurando-se a altura da cabeça em relação ao solo (AC), medindo-se a distância entre a região mentoniana da mandíbula e o chão, com o uso de uma régua afixada no tronco de contenção. Os valores totais registrados em centímetros foram convertidos em porcentagens, para evitar a variabilidade ocasionada pela diferença de tamanho entre animais.

O grau de analgesia promovido pelos tratamentos foi avaliado pela resposta a um estímulo nociceptivo induzido pela exposição rápida a um foco de luz de alta intensidade. O aparelho empregado para essa avaliação é formado por um processador que registra o tempo de exposição a um foco de luz emitido por uma fonte de luz artificial (Dolorimeter model 331 - IITC Inc. Life Science Instruments, USA), que gera uma temperatura de $140^{\circ} \mathrm{C}$. Ao direcionar-se o foco de luz para as regiões da coxa e coroa do casco, é possível apreciar a latência da resposta ao estímulo térmico, por movimentos de esquiva (LECC - latência do estímulo cutâneo da coxa) ou retirada do membro (LRRM- latência do reflexo de retirada do membro), de acordo com metodologia descrita ${ }^{18,19}$. A latência foi definida como o tempo que transcorreu entre a focalização do facho de luz e a reação do animal. A interrupção do estímulo doloroso ocorreu, invariavelmente, sempre que o tempo de exposição alcançasse 20 segundos, para evitar danos aos tecidos. A LRRM e a LECC sempre foram avaliadas pelo mesmo observador.
Os dados referentes às variáveis fisiológicas, comportamentais e nociceptivas aferidas foram tabelados e submetidos a análises de variância para repetições múltiplas One-Way Anova, seguidas de teste de StudentNewman-Keuls, para a avaliação das diferenças entre as médias ao longo do tempo, dentro de cada grupo. Para a comparação das médias, nos mesmos momentos, entre os grupos, foram utilizadas análises de variância sem repetições One-Way Anova, seguidas de teste de Student-Newman-Keuls.

\section{Resultados}

A administração epidural de amitraz (0,4 mg.kg-1 diluído com DMSO 10\% para um volume final de $5,0 \mathrm{~mL}$ ) promoveu vários graus de antinocicepção nas regiões da coxa e coroa do casco, que puderam ser demonstrados pelo aumento do tempo de latência da resposta ao estímulo nociceptivo (Figuras 1 e 2). Esse incremento da latência ocorreu entre os 25 e 120 minutos da injeção do fármaco, enquanto que nos outros grupos essa resposta não foi verificada. Com relação à FC, ambas as doses do amitraz provocaram redução dessa variável até os 240 minutos, em comparação aos valores basais e àqueles observados para o grupo controle. O amitraz inibiu a MR ao longo do período de observação, ao passo que o DMSO não interferiu nesse parâmetro. Os animais dos grupos amitraz 0,1 e controle permaneceram com a atividade de ruminação durante a fase experimental. Nos 3 grupos experimentais foi observada uma diminuição de no máximo $0,3^{\circ} \mathrm{C}$ na TR após cerca de 45 minutos, em comparação ao valor basal. Todas as vacas mantiveram-se em estação durante o experimento.

Alterações nas variáveis PAS, FR e AC não foram estatisticamente significativas $(p<0,05)$ entre os grupos nem dentro dos grupos em relação aos valores basais (Tabelas $1,2$ e 3$)$.

Os agonistas a-2 induzem analgesia ligando-se em estruturas sinápticas espinhais e supra-espinhais ${ }^{20}$ e, quando aplicados por 
Tabela 1 - Valores médios e desvios padrão das variáveis fisiológicas após injeção epidural de 5,0 mL de DMSO em vacas

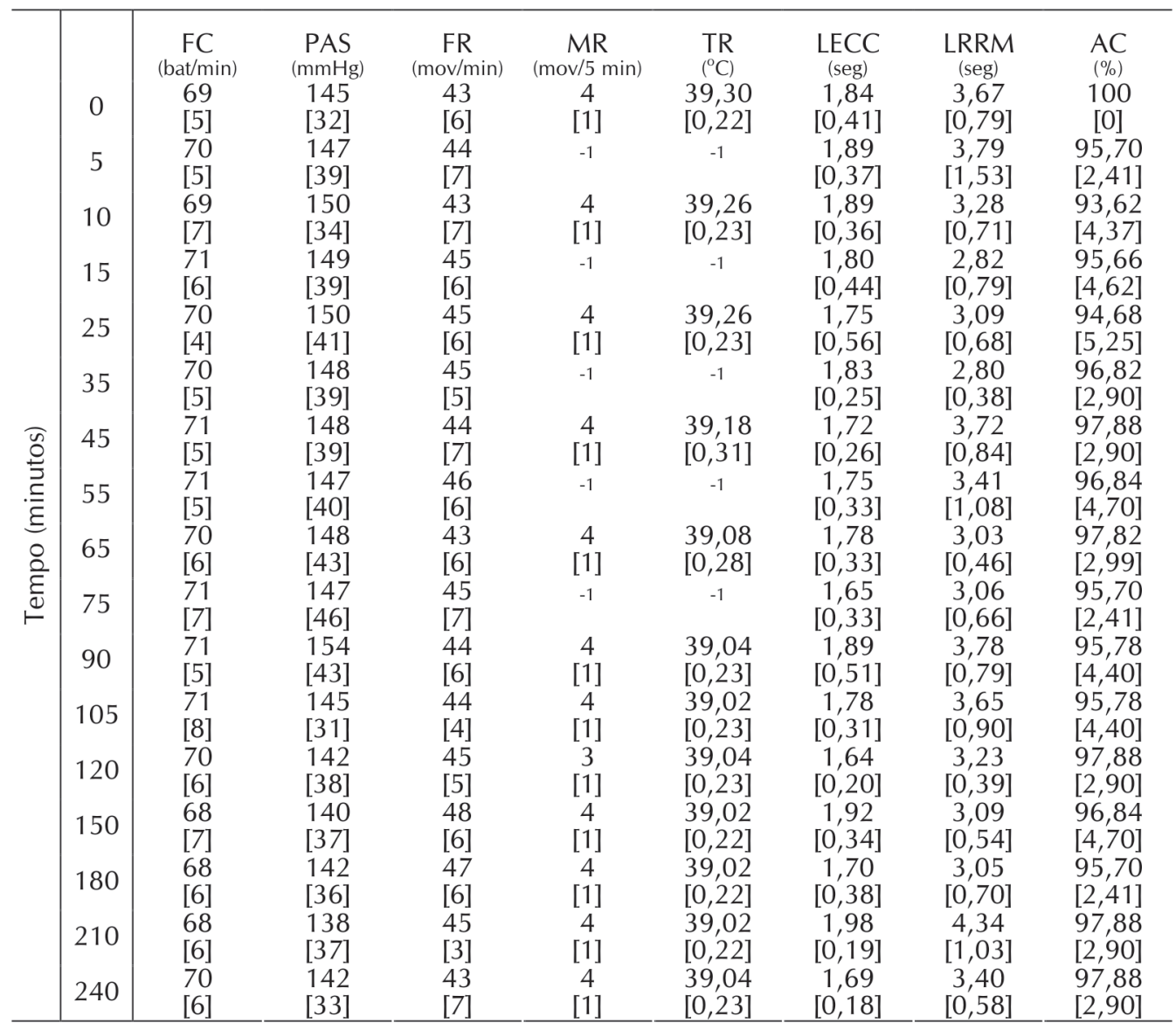

* Significativamente diferente de TO (teste Student-Newman-Keuls)

\# Significativamente diferente do grupo DMSO (teste Student-Newman-Keuls)

${ }^{-1}$ Dado não coletado

via epidural, agem em sítios receptores do corno dorsal da medula espinhal, inibindo a transmissão do estímulo nocivo mediado pela substância $\mathrm{P}^{21}$. A injeção epidural do agonista a-2 xilazina produziu antinocicepção em bovinos mediada pela interação com receptores espinhais, pois a administração sistêmica de antagonista a-2 não reverteu esse efeito $^{17}$. Esse mecanismo deve estar implicado no aumento da latência de resposta aos estímulos térmicos observados nos animais tratados com o amitraz na dose de $0,4 \mathrm{mg} \cdot \mathrm{kg}^{-1}$. Adicionalmente, deve ser considerada a possibilidade de uma relação dose-efeito, pois a dose de $0,1 \mathrm{mg} \cdot \mathrm{kg}^{-1}$ de amitraz não produziu efeito analgésico.
Nesse sentido, foi demonstrado que o amitraz, quando aplicado por via intravenosa em eqüinos, apresenta efeito antinociceptivo dose-dependente ${ }^{22}$. A administração epidural de DMSO não modificou a sensibilidade cutânea, de acordo com outros relatos na literatura $^{3,12,16}$.

Os valores médios da FC observados nos grupos tratados com amitraz permaneceram dentro da variação fisiológica para a espécie, no entanto, foram menores que os valores médios basais durante 240 minutos. A ocorrência de bradicardia seguida da aplicação epidural de agonistas a-2 foi descrita em bovinos ${ }^{6,7,17}$ e foi atribuída à inibição da liberação pré- 
Tabela 2 - Valores médios e desvios padrão das variáveis fisiológicas após injeção epidural de $0,1 \mathrm{mg} \cdot \mathrm{kg}^{-1}$ de amitraz em vacas

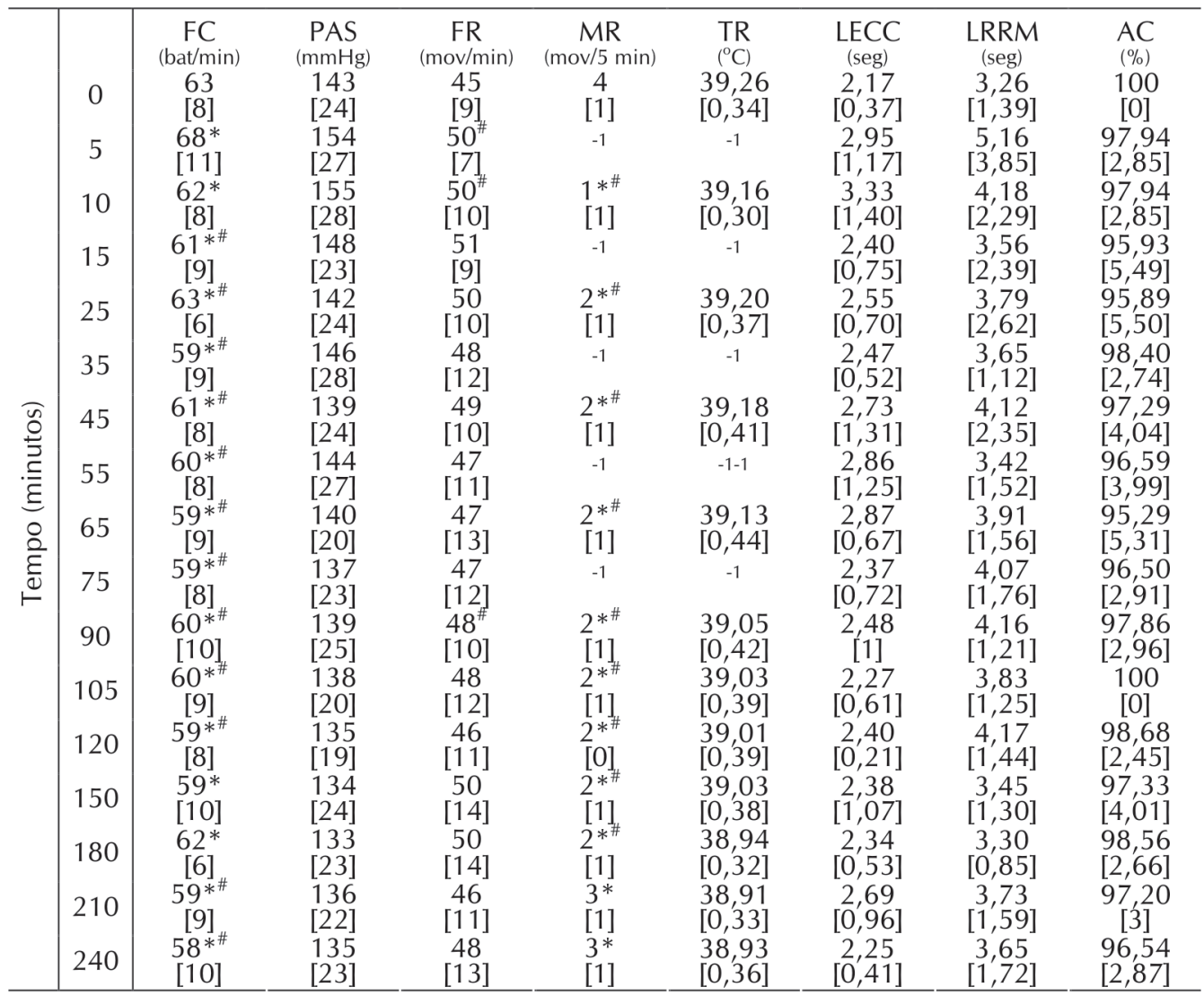

* Significativamente diferente de T0 (teste Student-Newman-Keuls)

\# Significativamente diferente do grupo DMSO (teste Student-Newman-Keuls)

${ }^{-1}$ Dado não coletado

sináptica de noradrenalina, diminuição do tônus simpático, liberação direta de acetilcolina no coração por fibras parassimpáticas e, principalmente, pelo aumento da atividade vagal reflexa em resposta à hipertensão transitória causada pela elevação inicial da resistência vascular periférica ${ }^{1,23}$. O mecanismo responsável pela redução da FC, observada nesse estudo, provavelmente foi a diminuição do tônus simpático, com conseqüente predominância da atividade vagal, pois o aumento da PAS só foi verificado nos animais tratados com a maior dose de amitraz, o que não justificaria a manifestação reflexa vagal apenas para esse grupo. O efeito vascular dos agonistas a-2 é caracterizado por hipertensão inicial seguida de hipotensão mais tardia. A elevação da pressão deve-se ao aumento da resistência periférica por vasoconstrição mediada pelos receptores a1 e a-2 localizados nos vasos sanguíneos, que causa aumento da resistência vascular periférica e, conseqüentemente, elevação da pós-carga fazendo com que haja bradicardia por ativação reflexa vagal. Posteriormente, há diminuição da pressão arterial, sustentada pela ação pré e pós sináptica central ou periférica dos agonistas a-2 nas terminações simpáticas ${ }^{23}$. Desse modo, o tratamento com a maior dose de amitraz provocou a elevação da PAS nos primeiros 15 minutos, enquanto a menor dose não alterou essa variável, de acordo com relatos em outras 
Tabela 3 - Valores médios e desvios padrão das variáveis fisiológicas após injeção epidural de 0,4 mg.kg¹ de amitraz em vacas

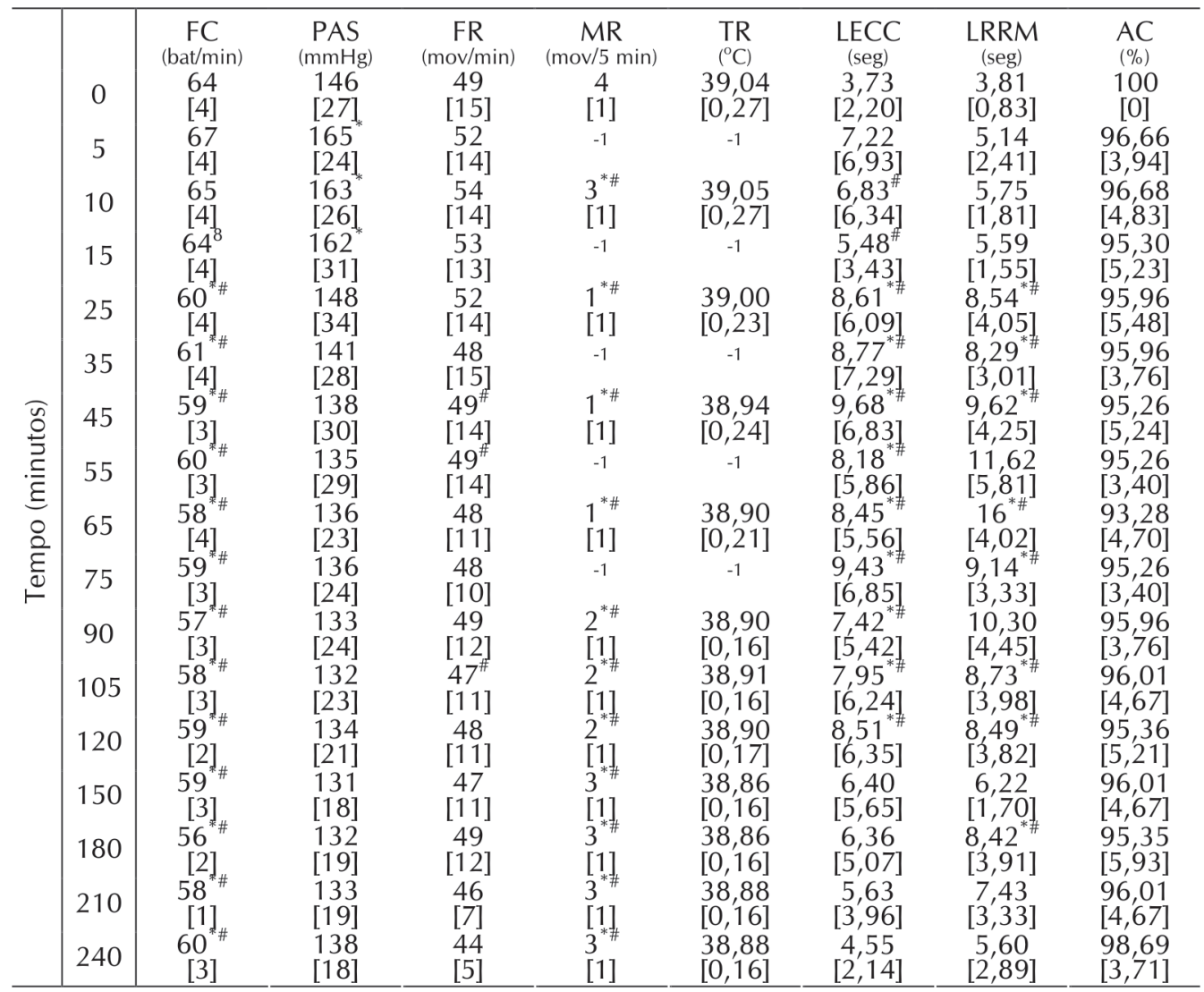

* Significativamente diferente de T0 (teste Student-Newman-Keuls)

\# Significativamente diferente do grupo DMSO (teste Student-Newman-Keuls)

${ }^{-1}$ Dado não coletado

espécies domésticas, como em eqüinos ${ }^{12}$, ou empregando-se outros fármacos agonistas a-2, em que a clonidina epidural não causou hipotensão em ovelhas ${ }^{3}$.

Os efeitos adrenérgicos a-2 sobre o sistema respiratório são citados como diminuição da FR e broncodilatação ${ }^{1}$ e são associados à inibição da atividade dos centros respiratórios superiores subseqüentemente à absorção sistêmica do agonista a-2 aplicado por via epidural ${ }^{6,7,17}$. Não foram verificadas alterações respiratórias nos animais tratados com amitraz, assim como em eqüinos, cujos parâmetros hemogasométricos de $\mathrm{PaO}_{2}, \mathrm{pH}$ e $\mathrm{PaCO}_{2}$ não sofreram modificações, o que permitiu supor que a função respiratória não foi alterada pela injeção epidural de amitraz ${ }^{12}$.
Os agonistas a-2 agem no sistema nervoso intrínseco mioentérico abaixando a concentração de acetilcolina na junção neuromuscular ${ }^{1,23}$. Os agonistas a-2 também diminuem prolongadamente a motilidade retículo-ruminal em bovinos, por bloqueio do mecanismo adrenérgico central que coordena a motilidade dos pré-estômagos ${ }^{24}$. Esses efeitos explicam a diminuição da MR nos grupos tratados com o amitraz. Os achados concordam com experimentos prévios realizados na espécie eqüina, nos quais a injeção intravenosa de $0,1 \mathrm{mg} \cdot \mathrm{kg}^{-1} \mathrm{de}$ amitraz reduziu a motilidade intestinal ${ }^{25}$. Ademais, na espécie bovina, a dose intravenosa de $0,4 \mathrm{mg} \cdot \mathrm{kg}^{-1}$, dessa substância resultou em efeitos inibitórios semelhantes 
aos da xilazina $\left(0,05 \mathrm{mg} \cdot \mathrm{kg}^{-1}, \mathrm{IV}\right)$ sobre a $\mathrm{MR}^{13}$.

Os agonistas a- 2 podem aumentar, reduzir ou não interferir na temperatura corpórea dessa espécie, porém, o mecanismo pelo qual essa classe de fármacos altera a termorregulação não foi completamente explicado na literatura ${ }^{6,17,26}$. No presente estudo, a diminuição da TR foi evidenciada após os diferentes tratamentos, sendo que todos os animais tiveram um decréscimo nos valores médios dessa variável. A explicação provável para a diminuição das temperaturas retais nos grupos amitraz e DMSO, que foi no máximo de $0,3{ }^{\circ} \mathrm{C}$ e se manteve dentro da faixa fisiológica para a espécie, pode ter sido que o experimento foi conduzido em sala climatizada, com temperatura que variou de 20 a $25^{\circ} \mathrm{C}$, pois o amitraz e o DMSO não induziram modificações nessa variável em outras ocasiões ${ }^{16,25}$

A sedação provocada pelos agonistas a-2 aplicados no espaço epidural resulta da absorção sistêmica e da conseqüente ação adrenérgica supra-espinhal ${ }^{21}$, demonstrada pela ausência de reversão do efeito sedativo da clonidina epidural por antagonista a-2 epidural, ocorrendo apenas reversão do efeito analgésico ${ }^{3}$. $\mathrm{O}$ abaixamento de cabeça que se observa durante a sedação resulta do relaxamento do tônus muscular do pescoço, semelhante ao que ocorre durante o sono ${ }^{27}$, sendo esse método já empregado para avaliar os efeitos sedativos de agonistas a-2 em bovinos $^{6,13,17}$. No entanto, a dose de amitraz empregada por via epidural não induziu sedação nos bovinos, provavelmente em função da dose, fato pode apoiar as poucas alterações observadas nas outras variáveis fisiológicas analisadas, como FR, PAS e TR.

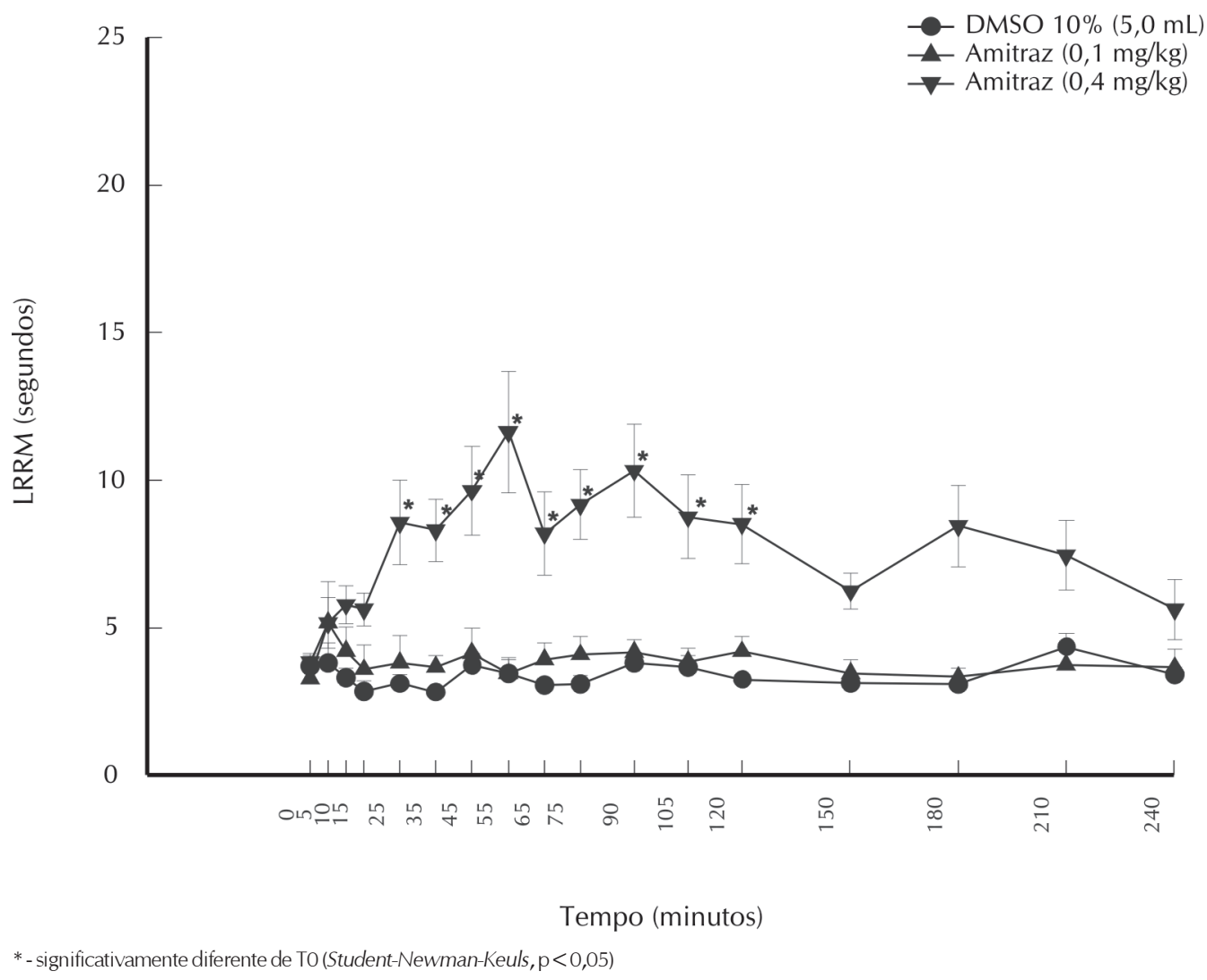

Figura 1 - Variação média da latência do estímulo cutâneo da coxa após injeção epidural de amitraz ou DMSO em vacas. Estão representadas as médias aritméticas e os erros padrão das médias 


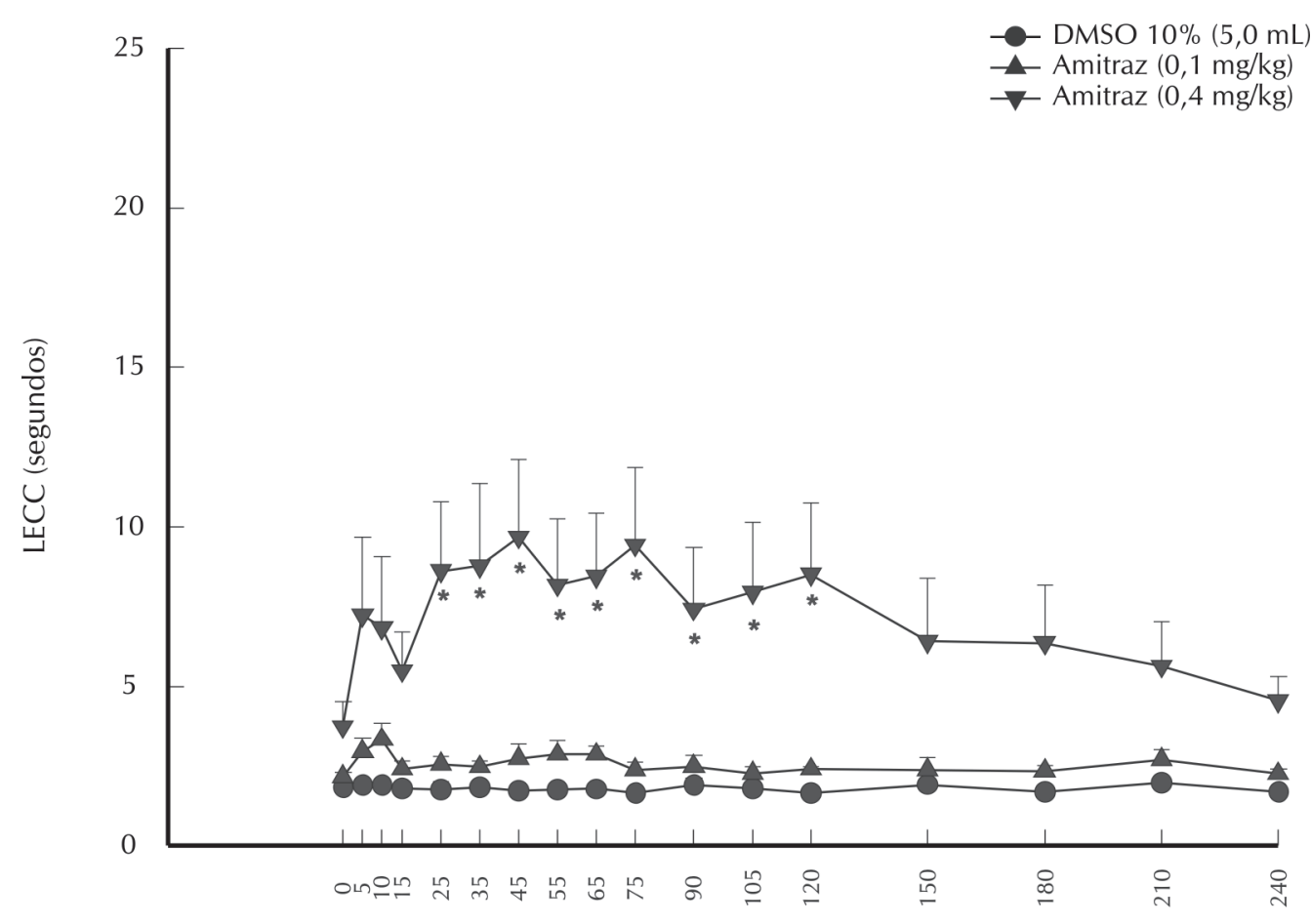

Tempo (minutos)

* -significativamente diferente de T0 (Student-Newman-Keuls, p<0,05)

Figura 2 - Variação média da latência do reflexo de retirada do membro após injeção epidural de amitraz ou DMSO em vacas. Estão representadas as médias aritméticas e os erros padrão das médias

\section{Conclusões}

Os resultados obtidos por meio da metodologia aplicada permitiram concluir que o amitraz, quando aplicado por via epidural, na dose de $0,4 \mathrm{mg} \cdot \mathrm{kg}^{-1}$, promove aumento da latência da resposta a um estímulo nociceptivo térmico e não causa efeitos colaterais sistêmicos de maior importância em vacas holandesas.

\section{Evaluation of the effects of epidural amitraz in cows}

\section{Abstract}

The amitraz is widely used in veterinary medicine as an acaricide in small and large animals. Several reports showed that the systemic effects caused by epidural administration of this substance are the result of its interaction with a-2 adrenoceptors. In the present study, the effects of epidurally amitraz $\left(0,1\right.$ or $\left.0,4 \mathrm{mg} \cdot \mathrm{kg}^{-1}\right)$ or $10 \%$ DMSO $(5,0 \mathrm{~mL})$ on the heart rate (HR), systolic arterial pressure (SAP), respiratory rate (RR), ruminal motility $(R M)$, rectal temperature $(R T)$, head height $(\mathrm{HH})$, and latency to heat-evoked reflexes (LSPR - skin of perineal region; LHWR - hoof withdrawal reflex) were evaluated. The HR and RM decreased in amitraz groups. There were no statistical differences in RR, SAP and HH among groups. LSPR and LHWR were prolonged in amitraz 0,4 group (T25-T120). We concluded that
Key-words: Alfa-2 agonist. Amitraz. Analgesia. Cattle. Epidural. 
epidural amitraz does not produce severe systemic effects, and that the higher dose induces prolongation of the latency to heat-evoked reflexes, in cows.

\section{Referências}

1 MAZE, M.; TRANQUILLI, W. Alpha-2 adrenoceptor agonists: defining the role in clinical anesthesia. Anesthesiology, Hagerstown, v. 74, n. 5, p. 581-605, 1991.

2 CARON, J. P.; LE BLANC, P. H. Caudal epidural analgesia in cattle using xylazine. Canadian Journal of Veterinary Research, Ottawa, v. 53, n. 4, p. 486-489, 1989.

3 EISENACH, J. C. et al. Epidural clonidine produces antinociception, but no hypotension, in sheep. Anesthesiology, Hagerstown, v. 66, n. 4, p. 496-501, 1987.

4 LE BLANC, P. H. et al. Epidural injection of xylazine for perineal analgesia in horses. Journal of American Veterinary Medical Association, Schaumburg, v. 193, n. 11 , p. $1405-1408,1988$.

5 RIEBOLD, T. W. et al. Comparison of lidocaine, xylazine, and lidocaine-xylazine for epidural anesthesia in cattle. Veterinary Surgery, Hagerstown, v. 21, p. 159, 1992.

6 ST. JEAN, G. et al. Caudal epidural analgesia induced by xylazine administration in cows. American Journa of Veterinary Research, Schaumburg, v. 51, n. 8, p. 1232-1236, 1990.

7 VESAL, N.; SAJEDIANFARD, J.; DEHGHANI, S. Epidural xylazine as na analgesic and sedative for teat surgery in cows. Journal of Applied Animal Research, v. 14 , p. $175-179,1998$.

8 SHARMA, S. P.; DABAS, Y. P. S. Therapeutic effects of dermocept and amitraz in clinical dermatological problems in dogs and calves. Indian Veterinary Journal, Madras, v. 70, n. 10, p. 952-955, 1993.

9 CULLEN, L. K.; REYNOLDSON, J. A. Central and peripheral alpha-adrenoceptor actions of amitraz in the dog. Journal of Veterinary Pharmacology and Therapeutics, Oxford, v. 13, n. 1, p. 86-92, 1990.

10 HSU, W. H.; LU, Z. X.; HEMBROUGH, F. B. Effects of amitraz on rate and aortic blood pressure in conscious dogs; Influence of atropine, prazosin, tolazoline and yohimbine. Toxicology and Applied Pharmacology, Orlando, v. 84, p. 418-422, 1986.

11 ROBERTS, M. C.; ARGENZIO, A. Effect of amitraz, several opiate derivatives and anticholinergic agents on intestinal transit in ponies. Equine Veterinary Journal, Newmarket, v. 18, n. 4, p. 256-260, 1986.

12 VALADÃO, C. A. A. Avaliação da injeção epidural do amitraz em eqüinos. 1998. 63 f. Tese (Livre Docência) - Faculdade de Ciências Agrárias e
Veterinárias, Universidade Estadual Paulista, Jaboticabal 1998.

13 POLIMENO, F. C. et al. Estudo dos efeitos do amitraz sobre parâmetros fisiológicos de bovinos. Ciência Animal Brasileira, Goiânia, v. 1, p. 210, 2000.

14 REIS, R. G.; ALMEIDA, R. M.; VALADÃO, C. A. A. Comparative evaluation of the association of xylazine or amitraz with ketamine at calves intravenous anesthetic induction. Archives of Veterinary Science, Curitiba, v. 6, p. 22, 2001.

15 JACOB, S. W. Current status of dimethyl sulfoxide (DMSO). Disponível em: < http://www.dmso.org/ articles/information/jacob.htm $>$. Acesso em: 25 nov. 2004.

16 BALESTRERO, L. T. Aspectos farmacológicos da injeção intravenosa ou epidural do amitraz, em eqüinos. 2001. 47 f. Dissertação (Mestrado) - Faculdade de Ciências Agrárias e Veterinárias, Universidade Estadual Paulista, Jaboticabal, 2001.

17 SKARDA, R. T.; ST. JEAN, G.; MUIR III, W. W. Influence of tolazoline on caudal epidural administration of xylazine in cattle. American Journal of Veterinary Research, Schaumburg, v. 51, n. 4, p. 556-560, 1990.

18 KAMERLING, S. G. et al. A method for studying cutaneous pain perception and analgesia in horses. Journal of Pharmacological Methods, New York, v. 13, p. 267-274, 1985.

19 PIPPI, N. L. et al. A model for evaluating pain in ponies. Journal of Equine Medicine Surgery, Princeton, v. 3, p. 430-435, 1979.

20 REDDY, S. V. R.; MADERDRUT, J. L.; YAKSH, T. L. Spinal cord pharmacology of adrenergic agonistmediated antinociception. Journal of Pharmacology and Experimental Therapeutics, Baltimore, v. 213, n. 3, p. 525-533, 1980

21 YAKSH, T. L. Pharmacology of spinal adrenergic systems which modulate spinal nociceptive processing. Pharmacology Biochemistry and Behavior, Tarrytown, v. 22, p. 845-858, 1985.

22 QUEIROZ NETO, A. et al. Characterization of the antinociceptive and sedative effect of amitraz in horses. Journal of Veterinary Pharmacology and Therapeutics, Oxford, v. 21, n. 5, p. 400-405, 1998.

23 DOHERTY, T. J. Physiologic effects of alpha two adrenergic receptors. Journal of American Veterinary Medical Association, Schaumburg, v. 192, n. 11, p. 1612-1614, 1988. 
24 RUCKEBUSCH, Y.; ALLAL, C. Depression of reticulo-ruminal motor functions through the stimulation of a2-adrenoceptors. Journal of Veterinary Pharmacology and Therapeutics, Oxford, v. 10, p. 1 10, 1987.

25 QUEIROZ NETO, A. et al. Effect of amitraz and xylazine on some physiological variables of horses. Arquivos Brasileiros de Medicina Veterinária e Zootecnia, Belo Horizonte, v. 52, n. 1, p. 27-32, 2000.

26 YOUNG, P. L. The effect of xylazine on the body temperature of cattle. Australian Veterinary Journal, Brisbane, v. 55, p. 442-443, 1979.

27 KAMERLING, S. G.; CRAVENS, W. M. T. BAGWELL, C. A. Objective assessment of detomidineinduced analgesia and sedation in the horse. European Journal of Pharmacology, v. 151, p. 1-8, 1988.

28 MUIR, M. DMSO: many uses, much controversy. Disponível em: < http://www.dmso.org/articles/ information/muir.htm >. Acesso em: 27 set. 2004. 